\title{
Rendimento, qualidade e longevidade de hastes de Photinia fraseri ${ }^{(1)}$
}

\author{
MARLISE NARA CIOTTA(2); EDUARDO DA COSTA NUNES ${ }^{(2)}$
}

\begin{abstract}
RESUMO
Os verdes de corte compreendem a parte aérea da planta sem a flor, mas com característica ornamental para a composição de arranjos. Na Serra Catarinense, uma espécie que tem se destacado é a Photinia fraseri, conhecida pelo nome comum de fotínia, que apresenta coloração diferenciada de suas folhas. O objetivo deste trabalho foi avaliar o rendimento de hastes e a durabilidade desta espécie em diferentes soluções com sacarose e temperatura. Foram coletadas, em duas épocas, 10 hastes em cada uma das alturas de corte (10,30 e 50cm), classificadas por tamanho, (CL1) maior ou igual a $60 \mathrm{~cm}$, (CL2) entre 60 e $45 \mathrm{~cm}$ e (CL3) menor que $45 \mathrm{~cm}$, e quantificadas. Para o teste de durabilidade, as hastes maiores que $60 \mathrm{~cm}$ foram submetidas a soluções aquosas de $0,5,10,15$ e $20 \%$ de sacarose por $24 \mathrm{~h}$, em duas temperaturas, e avaliadas quanto ao estádio de senescência (I ou II). O maior rendimento em número de hastes ocorreu na colheita realizada em março, relativamente à de novembro. As hastes mais longas, obtidas após o longo período de crescimento na colheita do mês de março, permitem ao lojista manusear e melhor utilizá-las em arranjos florais. Esta espécie apresenta um grande potencial em pós-colheita, uma vez que a longevidade das hastes com manutenção de sua qualidade ornamental é muito superior à maioria das plantas cultivadas como folhagem de corte.
\end{abstract}

Palavras-chave: hastes de corte, época de poda, durabilidade, pós-colheita.

\section{ABSTRACT \\ Yield, quality and longevity of stems of Photinia fraseri}

The greens understand cutting the shoots of the plant without the flower, but with ornamental feature to the composition of arrangements. In Sierra of Santa Catarina a species that has emerged is the Photinia fraseri, known by the common name of fotinia, which features distinctive color of its leaves. The aim of this study was to assess the yield of stems and durability of this species in solutions containing sucrose and temperature. Were collected during two periods, 10 stems in each of the cutting heights $(10,30$ and $50 \mathrm{~cm}$ ), classified by size, (CL1) greater than or equal to $60 \mathrm{~cm}$, (CL2) and between 60 and $45 \mathrm{~cm}$ (CL3) less than $45 \mathrm{~cm}$ and quantified. To test the durability stems greater than $60 \mathrm{~cm}$ were subjected to aqueous solutions of $0,5,10,15$ and $20 \%$ sucrose for $24 \mathrm{~h}$ at two temperatures and evaluated according to the stage of senescence (I, or II). The largest increase in number of stems at harvest was held in march in relation to november. The stems are longer, obtained after the long period of growth, harvest months of march and let the merchant handle best to use them in floral arrangements. This species has great potential in post-harvest, since the longevity of the stems with maintaining your ornamental quality is far superior to most plants grown as cut foliage.

Keywords: stems for cutting, pruning time, durability, postharvest.

\section{INTRODUÇÃO}

O setor da floricultura abrange diversos ramos de produção, alguns bastante especializados, permitindo bons rendimentos financeiros ao produtor.

Especialmente pela diversidade climática do Brasil, muitas espécies de flores e plantas ornamentais são possíveis de serem produzidas, o que tem contribuído muito para o crescimento do setor no País. Os últimos dados estatísticos do Instituto CEPA (2009) indicam a floricultura como importante atividade econômica no Brasil, com taxa de crescimento de $20 \%$ ao ano, sendo a participação das flores de corte no mercado nacional de $40 \%$. Com relação a produtos para exportação, uma pesquisa de campo realizada pelo Ibraflor (JUNQUEIRA et al., 2004) indica que as flores de corte representam $19,9 \%$ da área total destinada à floricultura. Dados de JUNQUEIRA e PEETZ (2007) também mostram o crescimento acentuado de folhagens e ramos cortados. Em 2007, o montante financeiro das exportações brasileiras representou uma participação percentual de $10,5 \%$ nas flores frescas, $41,9 \%$ nas mudas, $39,8 \%$ nos bulbos e $7,8 \%$ em produtos diversos.

As folhagens de corte compreendem uma série de produtos como folhas, ramos enfolhados, tanto herbáceos quanto lenhosos, ou seja, a parte aérea da planta sem a flor, mas com característica ornamental para a composição de arranjos.

Normalmente, algumas espécies, pela facilidade com que são encontradas em determinadas regiões, acabam sendo comumente utilizadas. Segundo OSHIRO (2001), alguns exemplos são o avencão (Rhumora adiantiformis), os aspargos ornamentias (Aspargus spp.), o eucalipto cinzento (Eucaliptus cineraea F. Muell), entre outros. No entanto, o mercado está constantemente buscando novidades, e a diversificação é importante e pode ser ainda muito estimulada.

\footnotetext{
(1) Recebido em 08/06/2011 e aceito para publicação em 1\%10/2012.

(2) Epagri/ Estação Experimental de São Joaquim, E.E.S.J., Rua João Araujo Lima, 102. Bairro Jardim Caiçara. C.P. 81, 88.600-000 São Joaquim, SC, fone (49) 3233-0324, e-mail: marlise@epagri.sc.gov.br; eduardon@epagri.sc.gov.br
} 
Na Serra Catarinense, uma espécie que tem se destacado é a Photinia fraseri, conhecida pelo nome comum de fotínia. O grande diferencial desta espécie é a intensa coloração avermelhada das folhas. As hastes novas apresentam folhas em sua extremidade de cor vermelha, que contrastam com o verde escuro das folhas mais velhas. Além da coloração, as folhas apresentam intenso brilho, o que possibilita o aproveitamento de qualquer haste da planta. A cor é responsável pelas sensações que produzem no observador e pelo efeito conferido ao arranjo, que pode variar da suavidade até a agressividade (KIKUCHI 1995). A cor avermelhada da fotínia promove um colorido contraste harmonioso em arranjos.

É uma espécie que pertence à família das Rosáceas, a mesma da rosa, flor já muito tradicionalmente usada em arranjos florais e cultivada em jardins. O clima ideal para seu desenvolvimento e para a intensidade de coloração avermelhada é o Sul do Brasil, nas regiões de altitude. Porém, a fotínia ainda é pouco conhecida e utilizada na floricultura.

Além do uso como haste fresca, também são importantes para a comercialização o período de conservação e a durabilidade de hastes cortadas para arranjos florais. Normalmente, buscando manter a maior longevidade de hastes, com qualidade, são utilizadas soluções aquosas contendo substâncias como açúcares, reguladores de crescimento e desinfetantes (germicidas), que auxiliam na manutenção da turgidez entre outras qualidades das hastes.

Desenvolveu-se este estudo com os objetivos de avaliar o rendimento de hastes de fotinia nas condições de cultivo da Serra Catarinense bem como conhecer a longevidade de hastes quando submetidas a diferentes concentrações de sacarose em condições de ambiente controlado (câmara fria) e não controlado (condições ambientais normais).

\section{MATERIAL E MÉTODOS}

Os trabalhos foram conduzidos na Estação Experimental da Epagri (Empresa de Pesquisa Agropecuária e Extensão Rural de Santa Catarina), em São Joaquim - SC.

\section{Experimento I - Rendimento e produção de hastes por classe de tamanho}

As plantas de fotínia utilizadas estão sendo cultivadas a campo, desde o ano de 2006, numa condição climática em que a temperatura média nos anos de 2006 e 2007 foi de $13,7^{\circ} \mathrm{C}$ e de $13,3^{\circ} \mathrm{C}$ em 2008 . Neste período de cultivo, as médias anuais pluviométricas foram de 1381, 1956 e 2052 mm para os anos de 2006, 2007 e 2008, respectivamente. O plantio ocorreu no mês de agosto, em espaçamento de $1,0 \mathrm{~m}$ entre linhas e $40 \mathrm{~cm}$ entre plantas, utilizando-se mudas obtidas de estaquia. Após o plantio, no ano seguinte foi iniciada a condução com podas realizadas no mês de março e novembro em três alturas em relação ao solo: 10 , 30 e $50 \mathrm{~cm}$. As hastes utilizadas nos experimentos foram coletadas de 10 plantas, logo após a poda, separadas em classes de tamanho, sendo maior ou igual a $60 \mathrm{~cm}$ (classe 1-CL1), entre 60 e $45 \mathrm{~cm}$ (classe 2- CL2) e menor que $45 \mathrm{~cm}$ (classe 3-CL3), e quantificadas (número de hastes por classe).

Os dados foram analisados utilizando-se a Análise de
Variância em uma direção (ANOVA), com separação de médias pelo DMS ao nível de significância de $5 \%(\mathrm{P}<0.05)$.

\section{Experimento II - Conservação pós-colheita de hastes}

Para este experimento, as hastes foram cortadas, selecionadas por tamanho (maior que 60cm) e posteriormente imersas (seis hastes por tratamento) em recipientes com $200 \mathrm{ml}$ de soluções aquosas de $0,5,10$, 15 e $20 \%$ de sacarose por $24 \mathrm{~h}$. Após este período, foram retiradas das soluções e mantidas em recipientes com 200 $\mathrm{ml}$ de água destilada somente, em duas diferentes condições ambientais: a) Sala com temperatura ambiente $\left(25^{\circ} \mathrm{C} \pm 2\right)$ e umidade em torno de $70 \%$ e b) Câmara fria com temperatura de $2^{\circ} \mathrm{C} \pm 2$, com ventilação/circulação de ar constante e umidade em torno de $90 \%$. As hastes permaneceram nestas condições até mostrar sinais evidentes do início da senescência. Foram avaliadas em relação à sua longevidade com manutenção de qualidade comercial, através de uma análise visual comparativa de qualidade, utilizando os critérios definidos a seguir: Estádio I, hastes apresentando e mantendo boa turgidez, rigidez da haste e folhas com cor intensa e brillante (padrão em ótimas condições de comercialização); Estádio II, hastes apresentando início ou ausência de turgidez da haste e perda da coloração característica da espécie, indicando neste caso o limite de qualidade mínino, considerando o seu valor ornamental e, consequentemente, comercial, ou seja, abaixo deste padrão, as hastes precisam ser descartadas. As plantas das quais foram colhidas as hastes para este experimento foram podadas no mês de novembro de 2008, portanto, as hastes submetidas aos tratamentos pós-colheita foram produzidas no verão, quando a intensidade de coloração das folhas é menor.

\section{RESULTADOS E DISCUSSÃO}

\section{Experimento I - Rendimento e produção de hastes por classe de tamanho}

Os resultados de rendimento estão apresentados nas Tabelas 1,2 e 3.

Os dados mostram diferenças no rendimento de hastes em função da sua época de corte. Na colheita realizada no mês de novembro, o total de hastes/planta foi, em média de 15, para as três alturas de poda das plantas (Tabela 1). Destas hastes, foram classificadas como CL1 60\% das hastes na altura de poda da planta de $50 \mathrm{~cm}$ (Tabela 3), bem como $80 \%$ e $75 \%$ nas alturas 30 e $10 \mathrm{~cm}$, respectivamente. Estes dados sao úteis para o produtor pois mostram a qualidade de hastes que podem ser produzidas nas condições de cultivo da Serra Catarinense. Para o mercado, normalmente, há maior interesse por hastes longas, que possibilitam o manuseio e o melhor aproveitamento delas na composição de arranjos.

$\mathrm{O}$ número total de hastes/planta no corte realizado em março variou de 25 a 30 (Tabela 2), no entanto, apenas em torno de $40 \%$ delas foram classificadas como CL1 e 47\%, CL2. O rendimento em relação à colheita de novembro foi significativamente maior, porém não proporcional à qualidade das hastes, o que pode implicar busca por 
mercados específicos para sua comercialização, limitando sua utilização como complemento em arranjos florais em que o tamanho da haste não é importante. Ou seja, esses resultados indicam que diferentes alturas de poda promovem produção de hastes de qualidade diferenciada, que também variaram segundo sua época de corte.

Trabalhos comparando épocas de corte de hastes foram desenvolvimos por SILVEIRA e MINAMI (1997) com a cultura do crisântemo. Estes autores observaram que, embora houvessem ocorrido hastes de diferentes dimensões, os dados observados para as quatro estações e em diferentes regiões foram relativamente uniformes.

BACKES et al. (2008), estudando diferentes tipos de poda em lisianto, observaram diferenças significativas. As plantas podadas acima do quinto par de folhas foram mais precoces em relação àquelas que receberam poda acima do terceiro par de folhas, recomendando a poda acima do quinto par de folhas.

Embora para a espécie deste trabalho não haja dados anteriores de outros autores, os diversos resultados com outras espécies ornamentais mostram que ocorrem diferenças entre época e/ou altura de corte.

Segundo NOORDEGRAAF (1994), o padrão de qualidade de flores e hastes de corte para o mercado é determinado por diferentes aspectos. Alguns destes aspectos podem ser mensurados através do comprimento da haste, tamanho da flor, número de flores, ou ainda pela visualização como forma, intensidade da cor e defeitos. Portanto, especialmente para uma espécie ainda sem padrão de qualidade estabelecido, caso da fotínia, torna-se fundamental obter parâmetros que indiquem características positivas para o mercado e para a comercialização.

\section{Experimento II - Conservação pós-colheita de hastes}

As hastes submetidas aos tratamentos com soluções aquosas de sacarose não mostraram ter seu período de longevidade alongado, independemente do ambiente em que se encontravam. O que significa dizer que não há efeitos positivos da sacarose no aumento da longevidade das hastes de $P$. fraseri (Figuras 2A e 2B). Resultados semelhantes foram obtidos em experimentos com a espécie Zinnia elegans, apresentados por CARNEIRO et al. (2002), em que a aplicação de sacarose não resultou na extensão da longevidade das flores, assim como acontece para outras espécies - ciclâme, estatice e orquídeas do gênero Oncidium - citadas pelo mesmo autor. HASTENREITER et al. (2006), avaliando pós-colheita de Oncidium varicosum, não observaram diferença na longevidade de flores utilizando diferentes tratamentos com "pulsing", seguidos de soluções de acondicionamento de sacarose.

Por outro lado, o efeito da sacarose pode ser diferenciado em hastes com flor. GONZAGA et al. (2001), trabalhando com inflorescências de girasol, observaram aumento da sua durabilidade quando submetidas a diferentes concentrações de sacarose, no entanto, quando a sacarose foi asociada a nitrato de prata, não houve resultados favoráveis na manutenção da qualidade comercial das inflorescencias. HAN (1992) afirma que a maioria das flores responde favoravelmente ao fornecimento de sacarose, mas o efeito "pulsing" de saturação dos tecidos com a sacarose depende muito da espécie e da concentração. TAIZ \& ZEIGER (2004) citam que o efeito do açúcar pode estar associado à sua translocação e a seu acúmulo nas flores, aumentando a concentração osmótica, melhorando a capacidade de absorção, favorecendo a manutenção da turgidez das pétalas e, consequentemente, o balanço hídrico das flores.

DIAS-TAGLIACOZZO et al. (2005) também observaram que o uso de uma solução de sacarose 4\% associada a $200 \mathrm{mg} / 1$ de ácido cítrico prolongou a durabilidade comercial de hastes florais de lírio em dois dias, em relação à solução somente com água.

Por todas estas referências, pode-se afirmar que as respostas a soluções conservantes sejam muito dependentes da espécie e, inclusive, da variedade. A concentração ótima de açúcares e o período de exposição à solução variam com o tratamento a ser utilizado e com a espécie a ser conservada (DIAS-TAGLIACOZZO et al., 2002).

Neste estudo com fotínia, por outro lado, houve diferença bastante significativa em relação à temperatura e umidade dos ambientes de armazenamento/conservação das hastes. Neste caso, as hastes mantidas em câmara fria apresentaram um período de longevidade superior quando comparado às hastes mantidas em condições de temperatura ambiente. Em câmara fria, as hastes mantiveram-se no estádio I até 61 dias em todos os tratamentos com ou sem imersão prévia em solução de sacarose. A partir deste período, começaram a surgir sintomas iniciais de murchamento das hastes, especialmente brotação apical, além de uma crescente e intensa descoloração e perda de brilho das folhas, que apresentaram bordas com manchas ou necrosadas (estádio II), conforme mostra a Figura 3. Em condições não controladas, temperatura ambiente, o período de durabilidade das hastes foi significativamente inferior, sendo que a senescência das hastes neste ambiente teve início a partir dos 38 dias de conservação.

Estes dados mostram o grande potencial de conservação da espécie em pós-colheita, uma vez que a longevidade é significativamente superior à maioria das plantas cultivadas como flor ou folhagem de corte. Esta é uma característica importante, pois possibilita armazenamento visando à venda estratégica em datas específicas ou de grandes volumes, na medida em que as hastes podem ser armazenadas por uma período relativamente longo, esperando melhores condições de comercialização, bem como viabilizando o transporte a mercados consumidores mais distantes em caminhões com sistema climatizado, reduzindo os riscos de perda de qualidades das hastes para comercialização.

SILVA et al. (2008) também observaram que a velocidade de surgimento de murcha, amarelecimento e necrose em hastes florais de gladíolos (Gladiolus grandiflora) se atrasou em temperatura de $10^{\circ} \mathrm{C}$ em relação à temperatura ambiente (aproximadamente $24^{\circ} \mathrm{C}$ ). Em temperaturas mais reduzidas, há menor mobilização de reservas e, consequentemente, ação de seus metabólitos.

\section{CONCLUSÕES}

O maior rendimento (número de hastes) ocorreu na colheita realizada em março.

As diferentes épocas de colheita resultaram na alteração da qualidade de hastes no que se refere ao seu comprimento. 
As hastes mais longas, obtidas na colheita do mês de março, permitem ao lojista manusear e melhor utilizá-las em arranjos florais.

Esta espécie apresenta um grande potencial de armazenamento pós-colheita, principalmente em condições controladas de câmara fria, com longevidade das hastes atingindo até 61 dias após o corte.

A espécie fotínia, pela sua longevidade pós-colheita em condições controladas de câmara fria, associada à sua beleza ornamental, apresenta grande potencial para ser explorada comercialmente.

\section{REFERÊNCIAS}

BACKES, F. A. A. L et al. Cultivo de lisianto para florde-corte sob diferentes tipos de poda. Bioscience Journal, Uberlândia, v.24, n.3, p.60-65, July/Sept., 2008.

CARNEIRO, T. F. et al. Influência da sacarose e do corte da base da haste na longevidade de inflorescências de Zinia elegans. Pesquisa Agropecuária Brasileira, Brasília, v.37, n.8, 2002.

DIAS-TAGLIACOZZO, G. D.; GONÇALVES, C. CASTRO, C. E.F. de. Fisiologia pós-colheita de espécies ornamentais. In: WACHOWICZ, C.M. e CARVALHO, R. I. N. (org.) - Fisiologia Vegetal: produção e pós-colheita, Curitiba: Champagnat, p.359-382, 2002.

DIAS-TAGLIACOZZO, G. D; GONÇALVES, C. CASTRO, C. E.F. de. Manutenção da qualidade póscolheita de lírio. Revista Brasileira Horticultura Ornamental, Campinas, v.11, n.1, p.29-34, 2005.

EMPRESA DE PESQUISA AGROPECUÁRIA E EXTENSÃO RURAL DE SANTA CATARINA - EPAGRI; CENTRO DE SOCIOECONOMIA E PLANEJAMENTO AGRÍCOLA - EPAGRI/CEPA. Síntese Anual da Agricultura de Santa Catarina 2008-2009. Disponível em: < http://cepa.epagri.sc.gov.br/Publicacoes/sintese_2009/ sintese_2009.pdf $>$ Acesso em 01 de fev. de 2010.

GONZAGA, A. dos R. et AL. Longevidade pós-colheita de inflorescências de girassol afetada por nitrato de prata e sacarose. Revista Brasileira Horticultura Ornamental, Campinas, v.7, n.1, p.73-77, 2001.
HAN, S. S. Role of sucrose in bud development and vase life of cut Liatris spicata (L.) Wild. HortScience, Massachusetts, v.27, n.11, p.1198-1200, 1992.

HASTENREITER, F. A. et al. Longevidade pós colheita de flores de Oncidium varicosum (Orchidaceae). Semina: Ciências Agrárias, Londrina, v.27, n.1, p. 27-34, 2006.

JUNQUEIRA, A. H.; PEETZ, M. da S. Pesquisa qualitativa sobre o potencial exportador de flores e plantas ornamentais do Brasil. Campinas: Ibraflor / APEX-Brasil: Programa FloraBrasilis, 2004, 1, CD-ROM.

JUNQUEIRA, A. H.; PEETZ, M. da S. Mercado interno para os produtos da floricultura brasileira: características, tendências e importância socioeconômica recente. Revista Brasileira Horticultura Ornamental, Campinas, v.14, n.1, p. 37-52, 2008.

KIKUCHI, O.Y. 1995. Ornamentação floral. São Paulo, Editora Senac, 73p.

NOORDEGRAAF, C.V. Production and marketing of high quality plants. Acta Horticulturae, Vertermate com Minoprio, v. 353, p. 134-138, 1994.

OSHIRO, L. et al. Comercialização e produção de folhagem ornamental de corte no Estado de São Paulo. Revista Brasileira Horticultura Ornamental, Campinas, v.7, n.1, p.55-73, 2001.

SILVA,L. R. da et al. Manejo pós-colheita de hastes florais de gladíolos (Gladiolus grandiflorus L.). Acta Agronomica, v.57, n.2, 2008.

SILVEIRA, R. B. de A. e MINAMI,K. Avaliação da qualidade de crisântemos (Dendranthema grandiflora Tzvelev.), grupo Macarrão, produzidos em diferentes regiões do Estado de São Paulo. Revista Brasileira Horticultura Ornamental, Campinas, v.3, n.2, p.1-8, 1997.

TAIZ, L.; ZEIGER, E. Fisiologia Vegetal. $3^{\text {a }}$ ed. Porto Alegre, Artmed, 2004. p. 61-91. 

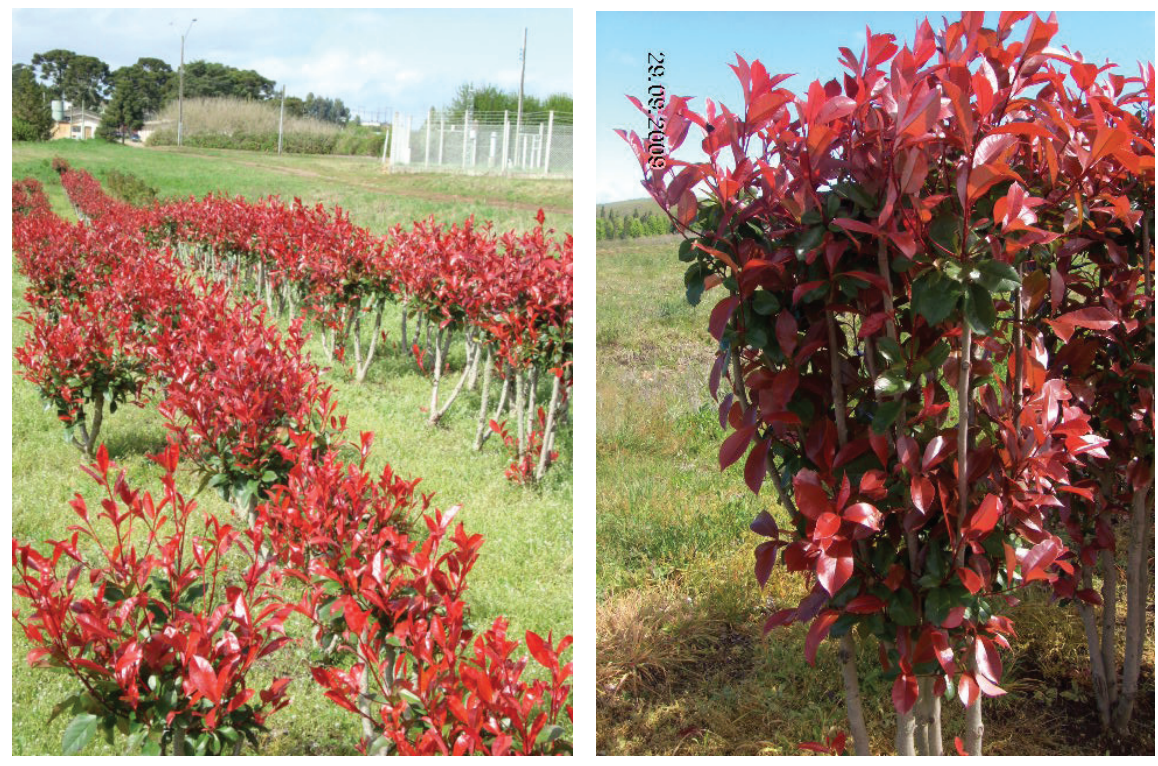

Figuras 1. (A e B). Plantas de Photinia fraseri a campo, após a poda (A) e cultivada como arbusto (B). Figures 1. (A and B). Plants of Photinia fraseri the field after pruning (A) and grown as a shrub (B).
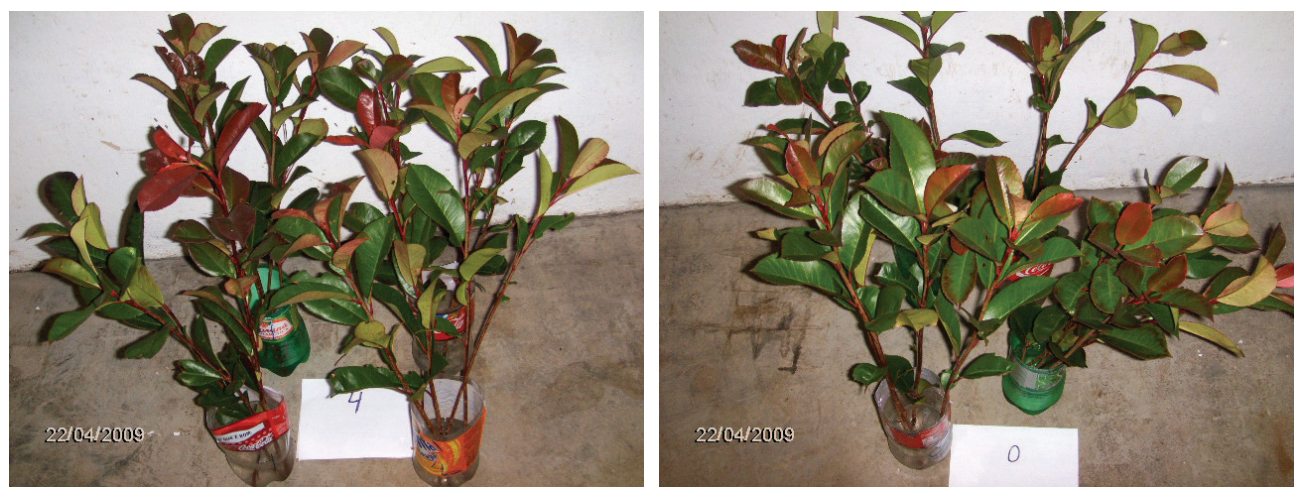

Figuras 2. (A e B). hastes de Photinia fraseri em câmara fria, em diferentes soluções de sacarose (1A 20\% de sacarose/24h e $1 \mathrm{~B}$ sem sacarose).

Figures 2. $(A$ and $B)$. Photinia fraseri stems in cold storage in different sucrose solutions $(20 \%$ sucrose $/ 24 h 1 A$ and $1 B$ without sucrose).

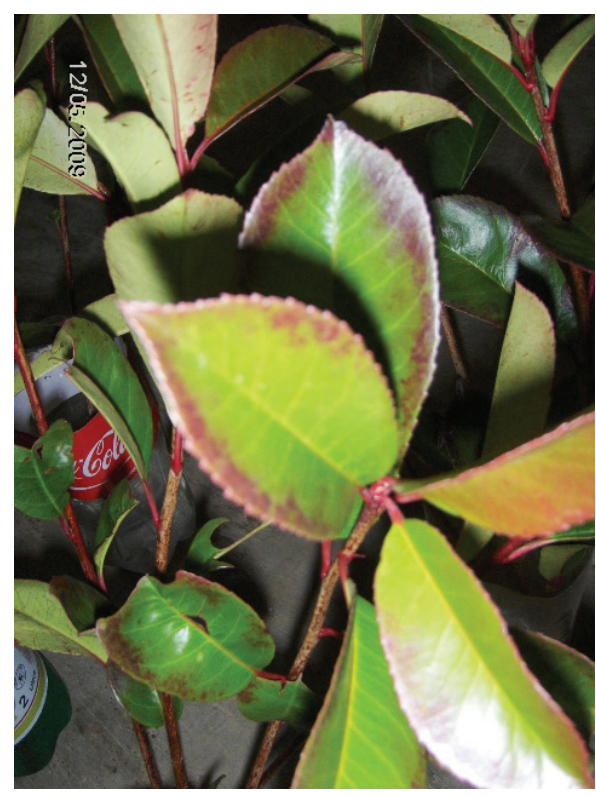

Figura 3. Folhas de Photinia fraseri mantidas em temperatura ambiente por 60 dias.

Figure 3. Leaves of Photinia fraseri kept at room temperature for 60 days. 
Tabela 1. Número médio de hastes para cada classe de tamanho, nas diferentes alturas de poda e colheita em novembro de Photinia fraseri

Table 1. Average number of stems for each size class at different times of pruning and harvesting in november $\underline{\text { Photinia }}$ fraseri

\begin{tabular}{ccccc} 
Altura de poda & \multicolumn{3}{c}{$\mathbf{N}^{\mathbf{0}}$ Hastes por classe/planta } & \multicolumn{2}{c}{ Total de hastes } \\
& Classe I-CL I & Classe II-CL II & Classe III-CL III & $15 \mathrm{a}$ \\
$\mathbf{5 0} \mathbf{~ c m}$ & $9 \mathrm{c}$ & $4 \mathrm{a}$ & $2 \mathrm{a}$ & $15 \mathrm{a}$ \\
$\mathbf{3 0} \mathbf{~ c m}$ & $12 \mathrm{a}$ & $2 \mathrm{a}$ & $1 \mathrm{a}$ & $16 \mathrm{a}$ \\
\hline $\mathbf{1 0} \mathbf{~ c m}$ & $12 \mathrm{a}$ & $3 \mathrm{a}$ & $1 \mathrm{a}$ & \\
\hline
\end{tabular}

Médias seguidas de mesma letra, na coluna, não diferem entre si ao nível de $5 \%$ de probabilidade.

Tabela 2. Número médio de hastes para cada classe de tamanho, nas diferentes alturas de poda e colheita em março de Photinia fraseri

Table 2. Average number of stems for each size class at different times of pruning and harvesting in march of $\underline{\text { Photinia }}$ fraseri

\begin{tabular}{ccccc} 
Altura de poda & \multicolumn{3}{c}{$\mathbf{N}^{\mathbf{0}}$ Hastes por classe/planta } & Total de hastes \\
& Classe I-CL I & Classe II-CL II & Classe III-CL III & $30 \mathrm{a}$ \\
$\mathbf{5 0} \mathbf{~ c m}$ & $13 \mathrm{a}$ & $14 \mathrm{a}$ & $3 \mathrm{a}$ & $25 \mathrm{a}$ \\
$\mathbf{3 0} \mathbf{~ c m}$ & $13 \mathrm{a}$ & $9 \mathrm{a}$ & $3 \mathrm{a}$ & $26 \mathrm{a}$ \\
\hline $\mathbf{1 0} \mathbf{~ c m}$ & $11 \mathrm{a}$ & $11 \mathrm{a}$ & $4 \mathrm{a}$ & \\
\hline
\end{tabular}

Tabela 3. Porcentagem de hastes/planta para cada classe de tamanho, nas diferentes alturas de poda de Photinia fraseri, nos meses de março e novembro, respectivamente

Table 3. Percentage of stems per plant for each size class at different times of pruning of Photinia fraseri in the months of march and november, respectively

\begin{tabular}{|c|c|c|c|c|}
\hline \multirow{2}{*}{ Época de poda } & \multirow{2}{*}{ Altura de poda } & \multicolumn{3}{|c|}{ (\%) Hastes por classe/planta } \\
\hline & & Classe I-CL I & Classe II-CL II & Classe III-CL III \\
\hline \multirow{3}{*}{ Março } & $50 \mathrm{~cm}$ & 43 & 47 & 11 \\
\hline & $30 \mathrm{~cm}$ & 52 & 36 & 12 \\
\hline & $10 \mathrm{~cm}$ & 42 & 42 & 16 \\
\hline \multirow{3}{*}{ Novembro } & $50 \mathrm{~cm}$ & 60 & 27 & 13 \\
\hline & $30 \mathrm{~cm}$ & 80 & 13 & 7 \\
\hline & $10 \mathrm{~cm}$ & 75 & 19 & 6 \\
\hline
\end{tabular}

